\title{
Automatic Accident Detection Techniques using CCTV Surveillance Videos: Methods, Data sets and Learning Strategies
}

\author{
Shilpa Jahagirdar, Sanjay Koli
}

\begin{abstract}
Intelligent communities are utilizing different creative ideas to improve the quality of human life. Due to fast growing sizes of our cities, need of travelling is constantly increasing, which in turn has increased count of vehicles on the roads. Increasing number of vehicles on the roads has brought about numerous difficulties for Street Traffic Management Authorities. Amongst different traffic related issues, road accidents are something worth giving attention to and have to be on the priority list. This paper describes various automatic road accident detection techniques, which automatically detect accidents using surveillance videos in real-time. As these methods do not consider various lighting conditions, changing weather conditions and different traffic patterns, none of the methods are robust enough to address all the incidences of the accident. In this paper, authors have described and compared many such methods.
\end{abstract}

Keywords: accident detection, CCTV, DNN, surveillance videos

\section{INTRODUCTION}

\section{A} ccording to the data provided by World Health Organization (WHO) in 2018, out of total road accidents in the world, $6 \%$ accidents take place in India where the number vehicles present on roads is only $1 \%$ of the world's total vehicles. In 2018, nearly 73\% of the deaths in South Asia region due to road accidents occurred in India. Also, according to World Health Organization (WHO)'s report in 2017, road accident is one of the most probable reason out of twelve reasons of death. It is also the ninth most common reason of early death, and the tenth most common cause for permanent disability [1]. Moreover, as per the report of UNESCAP, accidents are responsible for loss of GDP which is approximately $3 \%$ down every year in India due to road accidents [2]. According to recent statistics provided by the Ministry of Road Transport and Highways (India), in 2018 the number of overall road accidents in India was 4,67,044

Revised Manuscript Received on February 05, 2020.

* Correspondence Author

Shilpa Jahagirdar*, Research Scholar, Department of Electronics and Telecommunication, G. H. Raisoni College of Engineering and Management, Pune, India. Email: shipajahagirdar93@gmail.com

Dr. Sanjay Koli, Research Supervisor, Department of Electronics and Telecommunication, G. H. Raisoni College of Engineering and Management, Pune, India. And Professor, Department of E \& TC, Dr. D.Y. Patil School of Engineering, Charholi (Bk.), Pune, India. Email: sanjaykoli@yahoo.com

(C) The Authors. Published by Blue Eyes Intelligence Engineering and Sciences Publication (BEIESP). This is an open access article under the CC BY-NC-ND license (http://creativecommons.org/licenses/by-nc-nd/4.0/)

wherein 1,51417 people lost their lives and 4,69,418 faced severe injuries. And surprisingly, nearly $45 \%$ of the total road accidents took place on the roads other than national and state highways. These 'other' roads are the roads that are not properly planned and managed - usually found in urban areas [3]. This shows that the road accidents are something worth giving attention to and have to be on the priority list of the researcher's agenda to provide timely help at the place of accident and save lives, especially in the densely populated areas.

Many solutions to this problem have been attempted to build up a recognition strategy; however, the scope of these techniques is yet restricted to be practiced in real situations. Current solutions for automatic detection of accident can be classified as

1. Using additional hardware in vehicles. (sensors, modules, smart helmets etc.)

2. Using application on Smart phone and IoT. (using sensors from smart phone and transferring data to cloud)

3. Using existing CCTV surveillance video cameras.

Practically all the major urban communities over the world have already introduced noteworthy cameras for traffic checking. Making use of these already installed observation camera systems will be a feasible solution.[4]-[6].

Detecting road accidents automatically from the traffic surveillance CCTV video is an exceptionally attractive but challenging task. Over the years, research community as well as industry scientists have been attempting to develop a solution for automatic detection of accident using video processing techniques [7]-[10].

In this paper, section II describes related work on accident detection using video processing. Section III summarizes typical techniques implemented for accident detection in literature survey as well as comments about data set requirement. In Section IV, authors have illustrated various research challenges in this field. In section $\mathrm{V}$ authors discuss about the need of a new system for detecting an accident.

\section{LITERATURE SURVEY}

An innovative method inspired by motion of water waves to model the interaction among multiple moving objects for the detection of road accidents was developed by Kimin Yun et al. [11]. When many objects move on the water surface, the motion of water waves responding to them was the motivation for this method. 
By utilizing the symmetric properties of the MIF (Motion Interaction Field), traffic accidents are detected and localized. But this method considers only car accident cases. Data sets used for testing results were not made public for comparison. From the images, it can be observed that the less dense and lawful traffic videos were considered.

Furthermore, a novel method for real-time automatic detection of traffic accident was proposed [12]. Parameters extracted from the video frames form the basis of this system. Here, Gaussian Mixture Model (GMM) was used to detect the vehicles and mean shift algorithm was used to track the detected vehicles. After that, parameters were gathered from the video frames. The three significant parameters, the direction of the moving vehicles, the changes in the position of the vehicles and the speed of the vehicles were acquired to make the decision of accident. But various environmental and imaging conditions were not tested using proposed method. Data set used was very small and private.

Siyu Xia et al. used low-rank matrix approximation-based method for the detection of accident [13]. Approximation error was calculated for each block of frame and compared with defined threshold. Accidents were detected based on the comparison of approximation error with the defined threshold. Tested video segment was considered as normal, if the error was less than the threshold. Otherwise, it was labelled as a traffic accident. Similarly, a new detecting and positioning technique was addressed from the analysis of distribution characteristics based on average space occupancy, flow rate and average travel speed of traffic in a defined road segment [14]. In this method, every monitored lane segment was partitioned into a cluster of cells. By detecting and tracking traffic objects, parameters in each cell were acquired. This method was based on lawful traffic on highway. Accidents were detected based on congestion in a particular cell. Also, the data set used was private and hence, not available for comparison.

Additionally, an innovative method based on an adaptive traffic motion flow modelling technique was suggested [15]. In this method, Farneback Optical Flow was used for detection of motions and a statistic heuristic method was used for detection of accident. Threshold was varied in order to adapt changes in lighting conditions and traffic density. While testing results of the new system, only highway and expressway traffic patterns were considered. Database was small, private (self-created) and not available for comparison. Delay for processing video in this method is more so, it is not acceptable for real-time applications of accident detection and a 30 frame/sec camera.

Moreover, a new accident detection module was proposed that used different parameters like velocity, area, position, direction, and inclination angle of the moving object [16]. These parameters were extracted from the detected and tracked vehicles. Accident Index was calculated based on these parameters and compared with threshold to detect an accident. Incidences on highways and intersections were only considered. Authors suggested that the machine learning could be used to improve performance of the module. A private data set was used.

Perceptual video summarization technique was addressed for vehicle analysis and accident detection form traffic surveillance videos by Sinnu Susan Thomas and other co-authors [17]. Optimal summary-based event detection provides a faster visualization. However, depth-based segmentation, multi-camera scenario and varying environmental conditions can be considered to improve performance. No clear information was given about the exact data set used and was not made public for comparison.

Method introduced by Yuanlong Yu and other co-authors implemented three different modules: Low-level spatio-temporal feature, followed by sparse feature, and then W-ELM (Weighted-Extreme Learning Machine)-based detector [18]. The sliding window technique based on trained Kernel W-ELM classifier was used to determine whether each sliding window was a traffic accident scene or not. According to the author, there was a possibility of false detection in case of collision, and hence, more work was necessary in this issue. Enlargement of data set and supervised learning could be considered to improve the performance of the proposed system.

Object Detection and Tracking System along with a popular deep learning network was addressed by Kyu Beom et al. [19]. With the help of this method, it was possible to detect an accident within 10 seconds for each frame containing accident. But testing of method was done only for the particular case (tunnel videos) where traffic pattern was simple. Also, for testing author had used a very small data set of videos and data set was not made public for comparison.

For detecting accident, multi-modal deep learning method was used for the first time [20]. This method was verified on the real traffic from Hyderabad city in India. Accident detection rate of this method was about $77.5 \%$ and false alarms rate was $22.5 \%$ on real accidents videos. New data set (Indian Traffic) was made public for comparing results obtained from the other method. The challenges that need to be addressed in future are occlusions, poor visibility at night, and large variations in the normal traffic pattern.

\section{SUMMARY OF LITERATURE SURVEY}

\section{A. Techniques of accident detection}

Detection of road accident based on CCTV surveillance videos is mainly done by means of typical steps, which are motion detection, feature extraction, feature analysis, and accident recognition [15]. Typical steps for detecting accident in a video are shown in Figure 1.

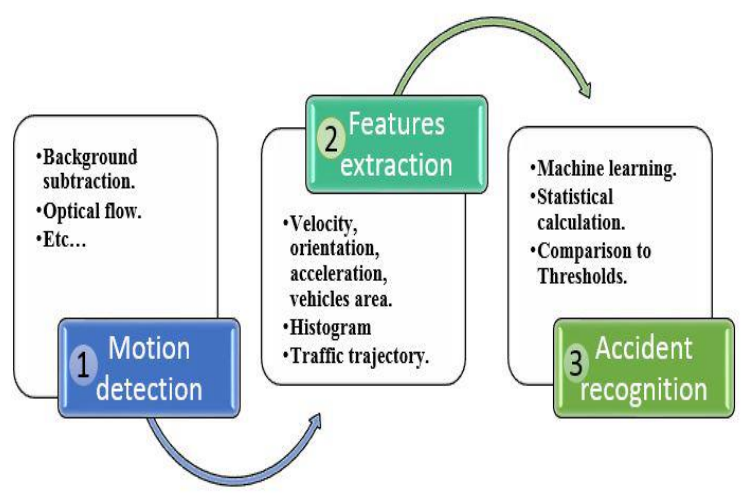

Fig. 1.Typical steps for accident detection[15].

Over the last few years, researchers have developed various systems to detect road accidents successfully [20]. The strategies used so far for detecting traffic accident from CCTV surveillance videos can be classified into three categories, namely modeling of traffic flow patterns, monitoring and analysis of vehicle activities and modeling of vehicle

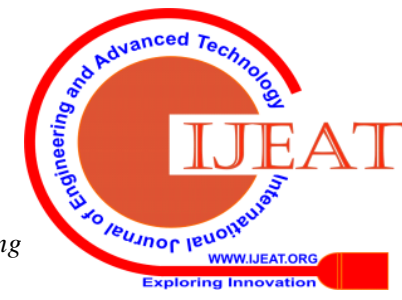


interactions. Most important factor in detecting accident is to track all the vehicles. Various current strategies utilize motion of moving vehicles to define a typical baseline (mostly using pre-decided threshold) and frame of any anonymous video, which does not follow the baseline is assumed as an accident.

The fact is, deviations from motion parameters provide useful information before collision, but it is not sufficient for accident detection. Furthermore, with a dense traffic and sudden change in the motion, the task becomes more challenging.

Thus, the existing methods to solve the problem of automatic detection have their own means to define "ACCIDENT", which may lead to FALSE DETECTION in real life. Difference between collision and occlusion needs more attention.

\section{B. Data set used for testing}

For developing effective accident detection system, collection of data set from CCTV surveillance videos is necessary. Data set must include videos of accident cases for various conditions as shown in Figure 2. Further, availability of public data set is also necessary so that other researchers can compare their results. Size of data set also matters as larger data set will be helpful to generalize results obtained during the research.
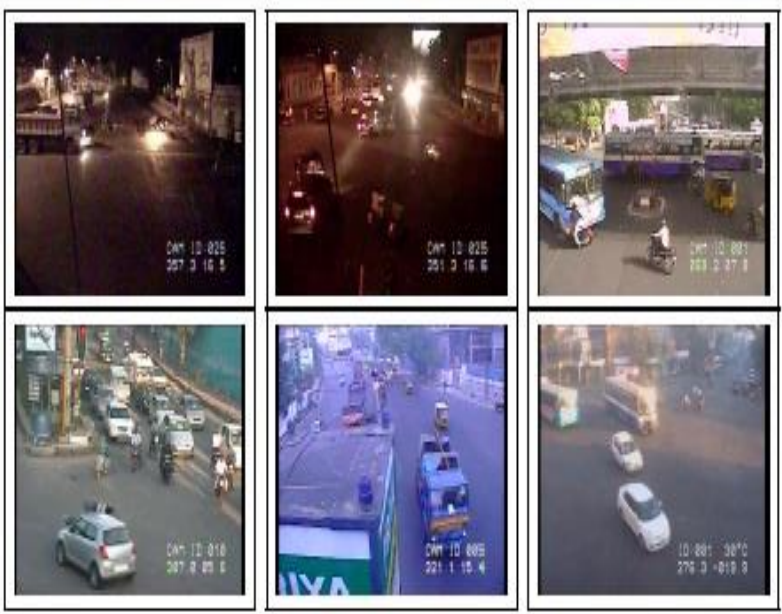

Fig. 2.Videos of accident that are recorded during various lighting conditions [20] [Best viewed in colour]

Unfortunately, data sets used in most of the methods for testing are either small in size, private and not available for comparison. Therefore, solutions suggested by these methods cannot be generalized. Furthermore, many of the methods are tested only on videos of lawful highway traffic or only on a particular type of cases [19]. Hence, these techniques may not be implemented for random traffic pattern. Various lighting and weather conditions are required to be considered for testing reliability of a system as they may change the performance of system in different conditions.

\section{CHALLENGES AND ISSUES}

After studying the literature on CCTV-based accident detection methods, different challenges and issues related to this area are identified.

Accident detection systems must have limited processing time so that these methods can be useful in real-time. Differentiating collision and occlusion is the most important factor that needs to be addressed particularly and it will help to reduce false accident detection rate. While developing such a system, different traffic patterns (highway/urban/random traffic) need to be considered. For developing more robust, generalized solution of the problem varied lighting conditions (day/night/evening/early morning) and different weather conditions (sunny/rainy/foggy) also need to be addressed. Availability of enough public data sets for comparing results with the work carried out earlier is also a major issue in this area.

\section{RESULTS AND DISCUSSION}

As public benchmark data set is not available, current techniques utilize a small private assortment of data sets. As data sets used are not publicly open, it is not reasonable to compare these methods. Still to understand the performances of various strategies discussed here, results of some methods are summarized in Table 1 with respect to criteria considered while designing the method.

Table 1. Criteria Considered And Accuracy By Various Methods

\begin{tabular}{|c|c|c|}
\hline Method & Criteria & Accuracy \\
\hline Kimin Yun [11] & $\begin{array}{c}\text { Only car accidents with law } \\
\text { full traffic and private data } \\
\text { set. }\end{array}$ & $89 \%$ \\
\hline J. Ren [14] & $\begin{array}{c}\text { Multilane (12) Highway only } \\
\text { with private data set. }\end{array}$ & $94 \%$ \\
\hline Boutheina Maalouly [15] & $\begin{array}{c}\text { Highways and Expressways } \\
\text { only with private data set. }\end{array}$ & $77 \%$ \\
\hline Sinnu Susan [17] & $\begin{array}{c}\text { Data sets from You tube and } \\
\text { Urban Tracker }\end{array}$ & $82 \%$ \\
\hline Yuanlong Yu [18] & Data set from You tube & $>90 \%$ \\
\hline Dinesh Singh [20] & $\begin{array}{c}\text { Indian Real Traffic videos } \\
\text { from Hyderabad city with } \\
\text { new public data set. }\end{array}$ & $77.5 \%$ \\
\hline
\end{tabular}

\section{CONCLUSION}

Different methods for automatically detecting an accident with the help of CCTV surveillance videos are discussed and compared in this paper. In spite of exceptionally advisable task, very less and restricted work is carried out in this field. Some methods are developed to work on particular pattern of traffic in restricted environment and those do not consider other affecting factors. All these strategies can said to be reasonable for small sized samples and don't ensure the same performance in different situation. This creates an urge to design a new system for automatically detecting accident addressing lawful as well as lawless traffic patterns in various illumination and different environmental conditions and to compare results on some common data set.

\section{ACKNOWLEDGMENT}

The authors would like to thank Chalavadi Krishna Mohan, Member IEEE, Associate Professor, Department of Computer Science and Engineering, IIT Hyderabad, India and Dinesh Singh, Student Member IEEE, Assistant Professor, Department of Computer Science and Engineering, IIT Hyderabad, India for the data set made available from the CCTV surveillance network of Hyderabad city in India, which is made public for the research community for further comparison. 


\section{REFERENCES}

1. World Health Organization (WHO), "Global status report on road safety 2018 ”, https://www.who.int/news-room/fact-sheets/detail/ road-traffic-injuries.

2. Ministry of Road Transport and Highways, Government of India, "Road accidents in India, issues and dimensions", https://www.unescap.org/sites/default/files/2.12.India_.pdf.

3. Government of India, "Road accidents in India", https://morth.nic.in/sites/default/files/Road_Accidednt.pdf, 2018.

4. S. Djahel, R. Doolan, G.-M. Muntean, and J. Murphy, "A communications-oriented perspective on traffic management systems for Smart Cities: Challenges and innovative approaches", IEEE Communication. Surveys Tutorials, Volume: 17, Issue 1, pp.125-151, March 2015.

5. European Initiative on Smart Cities, 2010-2020. Accessed: May 15, 2018. [Online]. Available: https://setis.ec.europa.eu/set-planimplementation/ technology-roadmaps/european-initiative-smart-cities.

6. V. Kostakos, T. Ojala, and T. Juntunen, "Traffic in the Smart City: Exploring city-wide sensing for traffic control center augmentation", IEEE Internet Computing, Volume: 17, Issue 6, pp. 22-29, November 2013.

7. C. Regazzoni, A. Cavallaro, Y. Wu, J. Konrad, and A. Hampapur, "Video analytics for surveillance: Theory and practice", IEEE Signal Processing Magazine, Volume: 27, Issue 5, September 2010.

8. G. Yuan, X. Zhang, Q. Yao, and K. Wang, "Hierarchical and modular surveillance systems in ITS", IEEE Intelligent System, Volume: 26, Issue 5, pp.10-15, October 2011.

9. A. Dopfer and C.-C. Wang, "What can we learn from accident videos?", Proceedings of International Automatic Control Conference (CACS), December 2013.

10. I. Celino, S. Kotoulas, "Smart cities [Guest editors' introduction]", IEEE Internet Computing, Volume: 17, Issue 6, pp. 8-11, December. 2013.

11. Kimin Yun, Hawook Jeong, Kwang Moo Yi, Soo Wan Kim, Jin Young Choi, "Motion Interaction Field for Accident Detection in Traffic Surveillance Video", IEEE, 22 ${ }^{\text {nd }}$ International Conference on Pattern Recognition, December 2014.

12. Zu hui, Xie yaohua, Ma lu,Fu Jiansheng, "Vision-based real-time traffic accident detection", IEEE, Proceedings of $11^{\text {th }}$ World Congress on Intelligent Control and Automation, July 2015.

13. Siyu Xia, Jian Xiong, Ying Liu and Gang Li, "Vision-based Traffic Accident Detection Using Matrix Approximation", IEEE, $10^{\text {th }}$ Asian Control Conference (ASCC), June 2015.

14. J. Ren, Y. Chen, L. Xin, J. Shi, B. Li, and Y. Liu, "Detecting and positioning of traffic incidents via video-based analysis of traffic states in a road segment", IET Intelligent Transport Systems, Volume: 10, Issue 6, pp. 428-437, August 2016.

15. Boutheina Maalouly, Abdelmalik Taleb-Ahmed, Smail Niar, Naim Harby and Carlos Valderramay, "Adaptive Video-Based Algorithm for Accident detection on Highways", proceedings of IEEE International Symposium on Industrial Embedded Systems (SIES), 2017.

16. Gargi Desai, Sagar Jakharia, Vishal Ambre, Snehal Sherkhane, "Smart Road Surveillance Using Image Processing", International Conference on Smart City and Emerging Technology, ICSCET, January 2018.

17. Sinnu Susan Thomas, Sumana Gupta, Venkatesh K. Subramanian, "Event Detection on Roads Using Perceptual Video Summarization", IEEE Transactions on Intelligent Transportation Systems, Volume: 19, Issue 9, pp. 2944-2954, September 2018.

18. Yuanlong Yu, Miaoxing Xu, Jason Gu, "Vision-based traffic accident detection using sparse spatio-temporal features and weighted extreme learning machine", IEEE, IET Intelligent Transport Systems, Volume: 13, Issue 9, pp. 1417-1428, September 2019.

19. Kyu Beom Lee, Hyu Soung Shin, “An application of a deep learning algorithm for automatic detection of unexpected accidents under bad CCTV monitoring conditions tunnels", IEEE, International Conference on Deep Learning and Machine Learning in Emerging Applications (Deep-ML), 2019.

20. Singh and Chalavadi Krishna Mohan, "Deep spatio-temporal representation for detection of road accidents", IEEE Transactions on Intelligent Transportation Systems, Volume: 20, Issue: 3, pp.879-887, March 2019.

\section{AUTHORS PROFILE}

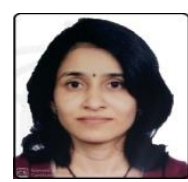

Shilpa Jahagirdar has completed her Bachelor and Master's degree in Electronics Engineering from S.G.G.S.C.O.E.T., Nanded, Dr. Babasaheb Ambedkar Marathwada University, India. Currently she is perusing her Ph.D. from G.H. Raisoni College of Engineering and Management, Pune, India. She works as an Assistant Professor in Smt. Kashibai Navale College of Engineering, Pune, India. Her research interest is video signal processing.

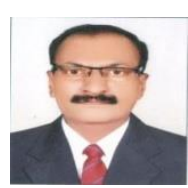

Dr. Sanjay Koli has received his B.E. degree from the Department of Electronics Engineering from Shivaji University, Kolhapur, Maharashtra, India in 1997, M. Tech. degree from the Department of Electronics and Telecommunication Engineering from Dr. Babasaheb Ambedkar Technological University, Lonere, Maharashtra, India in 2006 and Ph.D. degree from Department of Engineering and Technology, Sant Gadage Baba Amravati University, Amravati, Maharashtra, India in 2015. He is currently working as a Professor in the Department of Electronics and Telecommunication Engineering at Dr. D. Y. Patil School of Engineering, Pune, India and is a research supervisor at G. H. Raisoni College of Engineering and Management, Pune, India. His research interests mainly include wireless domain and video signal processing. 